'Departamento de Psiquiatría y Salud Mental Oriente. Facultad de Medicina Universidad de Chile. Santiago, Chile.

${ }^{2}$ Centro Comunitario de Salud Mental (COSAM) Peñalolén. Santiago, Chile.

${ }^{3}$ Unidad de Psiquiatría de Enlace Hospital Santiago Oriente Doctor Luis Tisné Brousse. Santiago, Chile.

No hubo apoyo financiero de ningún tipo para la preparación de este trabajo de investigación. Los autores declaran no tener conflicto de intereses.

Recibido el 11 de julio de 2017 , aceptado el 6 de febrero de 2018.

Correspondencia a: Juan C. Almonte Obispo Donoso 24, dpto. 4, Providencia. Santiago, Chile. jcalmonte@gmail.com

\section{Licencia médica psiquiátrica: revisión de los efectos positivos y negativos del reposo}

\author{
JUAN C. ALMONTE ${ }^{1}$, PABLO MUÑOZ ${ }^{2,3}$, JAVIERA PARRINI $^{3}$
}

\section{Psychiatric sick leave}

There is no consensus amongst physicians, social security representatives and researchers about optimum sick leaves. This is an indication that should maximize positive outcomes and minimize potential side effects, both for the patient and society. The use of sick leaves during the last decade rose steadily, particularly in the psychiatric field. The most important causes of this increase are: changes in public policies, overuse of psychiatric diagnosis to cover up unmet social needs, and modifications to labour structure. It is analysed the impact that this situation has implied for physician patient relationship as well as for health budget. Even though sick leave diminishes presentism associated to a psychiatric disorder, published evidence about the effect of prolonged sick leave shows that damage overruns potential benefits: augmented morbidity and mortality, workplace phobia, economic loss, among others.

(Rev Med Chile 2018; 146: 494-501)

Key words: Mental Health; Psychiatry; Sick Leave.

\section{Contexto}

1. Aumento de la carga de salud asociada a patología psiquiátrica: tendencia mundial y nacional

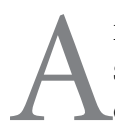
nivel de salud global, la patología mental se ha establecido como la principal causa de discapacidad tanto transitoria como permanente ${ }^{1}$. En Chile, el gasto total en licencias médicas ha aumentado en más de $100 \%$ entre los años 2001 y $2015^{2,3}$. Son múltiples los factores que aquí intervendrían: cambios en el modo de diagnosticar, desarrollo de políticas públicas que mejoran el acceso a servicios de psiquiatría, modificaciones en determinantes sociales relevantes para la salud mental, por nombrar algunos.

En las décadas de 1950-59 y 1960-69 se empieza a gestar en Chile un cambio de paradigma desde una psiquiatría asilar a una ambulatoria, primero a través de la implementación de servicios de psiquiatría en hospitales generales y luego a través de diversos programas dedicados al tratamiento de trastornos mentales ${ }^{4}$. Los planes nacionales de Salud Mental de los años 1993 y $2000^{5,6}$ dieron un nuevo impulso a la psiquiatría comunitaria y ambulatoria a través del aumento y redistribución del gasto destinado a salud mental. Hoy existe un mejor acceso a servicios de salud mental y, en general, la patología tratada es de menor gravedad. Esto significa que se atiende a una población con mayor funcionalidad sociolaboral que antes. Tal escenario sitúa, por tanto, con mayor frecuencia al médico ante la tarea de determinar cuándo una persona deja de estar apta para trabajar. Los recursos asociados a la atención psiquiátrica han tenido entonces que destinarse progresivamente al pago de licencias médicas. Por ejemplo, en Suecia, en el año 2005, 85\% del gasto que significó al Estado el manejo de la depresión correspondió a compensaciones económicas por reposo médico y pensiones por jubilación anticipada ${ }^{7}$.

En la Tabla 1, se exponen datos de los años 2004 al 2015 que resumen la magnitud de la indicación de reposo médico por causa psiquiátrica 
Tabla 1. Carga de la patología psiquiátrica en Chile $2,3,47,56$

\begin{tabular}{|ll|}
\hline Porcentaje de AVISA por causa psiquiátrica respecto al total (2004) & $27,6 \%$ \\
\hline Porcentaje de licencias psiquiátricas respecto al total (Fonasa e Isapre, 2015) & $22 \%$ \\
\hline Porcentaje del gasto por licencias psiquiátricas respecto al total (Fonasa e Isapre, 2015) & $27 \%$ \\
\hline Gasto total por licencias psiquiátricas (Fonasa e Isapre, 2015) & $\$ 263.702$ Millones \\
\hline Duración promedio por licencia psiquiátrica (Fonasa e Isapre, 2015) & 16,5 días \\
\hline Duración promedio del reposo psiquiátrico total por persona (Fonasa, 2008) & 98 días \\
\hline Gasto promedio por licencia psiquiátrica (Fonasa, 2015) & $\$ 255.966$ \\
\hline Gasto promedio por licencia psiquiátrica (Isapre, 2015) & $\$ 361.707$ \\
\hline Porcentaje de licencias psiquiátricas conducentes a invalidez (Fonasa, 2008) & $0,6 \%$ \\
\hline Diagnóstico más frecuente en licencias psiquiátricas (Fonasa, 2008) & Depresión (70\%) \\
\hline
\end{tabular}

en Chile. Durante el período contemplado destaca que la patología psiquiátrica alcanza el primer lugar del total de licencias indicadas $(27 \%$ del gasto total del país destinado a reposo médico) y que la duración promedio de reposo psiquiátrico por persona sea de 98 días. Considerando que el reposo médico representa una problemática compleja, no solo por los altos gastos asociados a este, sino también por las conflictivas psicosociales que podrían subyacer, surge el interés por entender más profundamente este fenómeno, en vista a una adecuada gestión de políticas públicas.

\section{Rol del reposo médico ante el malestar social}

Junto a los planes nacionales de salud mental, otra política reciente que ha impactado en el acceso a la atención de salud mental ha sido el Plan de Garantías Explícitas en Salud (GES). Incluir la depresión como uno de los diagnósticos de atención priorizada ha determinado un aumento significativo en las consultas psiquiátricas. Al garantizar el acceso oportuno a terapéuticas para este trastorno, la consulta psiquiátrica se ha vuelto masiva y expedita. Sin embargo, uno de los problemas con la depresión como diagnóstico es que esta corresponde a un constructo heterogéneo y de márgenes laxos ${ }^{8,9}$, como lo indica, por ejemplo, que el modo de diagnosticar cambie ampliamente entre distintos grupos de médicos ${ }^{10}$, a diferencia de enfermedades médicas no psiquiátricas.

Debemos considerar también cambios del entorno laboral de las últimas décadas (crecimiento del área de servicios, informalidad y externalización de contratos, incorporación de tecnología compleja, entre otros), que determinan una mayor exposición a estrés sostenido en el tiempo ${ }^{11}$. A esto se suma que Chile es el $5^{\circ}$ país de la Organización para la Cooperación y el Desarrollo Económico (OCDE) con jornadas de trabajo más largas ${ }^{12}$. Tales factores determinan una elevación del malestar psicosocial, el que eventualmente puede aumentar el riesgo de patología mental y, por tanto, de mayor consulta psiquiátrica. En estos casos, la indicación de reposo médico se ve reforzada por el hecho de que este permitiría, al menos transitoriamente, manejar uno de los posibles factores de riesgo en cuestión, aunque privilegiando una estrategia evitativa.

Al tomar en cuenta los factores hasta aquí planteados, se puede pensar que el amplio solapamiento que existe en términos diagnósticos entre reacciones vivenciales normales y anormales (entre ellas la depresión clínica) facilita el uso de la "vía psiquiátrica" para resolver problemas de índole más bien ambiental ${ }^{13}$. Existe una carencia de políticas de bienestar social que faciliten el enfrentamiento de estresores psicosociales habituales, como podría ser el caso de la enfermedad de un familiar. Todo esto genera condiciones propicias para que la depresión sea objeto de sobrediagnóstico.

\section{Ausencia de directrices para la indicación del reposo médico}

Estudios en distintos países han reportado que los médicos encuentran problemática la indicación de reposo laboral ${ }^{14,19}$. Una dificultad frecuentemente citada es el conflicto de lealtades al asumir 
un "doble rol", no solo como médico del paciente, sino también como profesional experto ante las aseguradoras de salud y los empleadore ${ }^{20}$. Esto se agrava al considerar que la evidencia que pudiese respaldar esta práctica clínica es muy limitada ${ }^{21}$.

Desde un punto de vista estrictamente normativo (i.e. marcos legislativos internacionales), el tipo y gravedad de una enfermedad médica, así como la limitación funcional que esta produce, deberían ser los principales criterios para determinar la indicación de reposo ${ }^{22-24}$, sin embargo, esta puede responder a otras causas más allá de una enfermedad (Tabla 2). En la práctica, la determinación de incapacidad laboral es el resultado de un complejo proceso de evaluación en el cual el médico, a partir de diversas fuentes de información, trata de balancear la clínica con las circunstancias sociolaborales del paciente. Ponderar el impacto que una enfermedad mental genera en la funcionalidad de un paciente es una tarea compleja y que difícilmente se puede abarcar en el contexto de una consulta médica.

\section{Efectos iatrogénicos y terapéuticos del reposo médico en salud mental}

\section{Efectos positivos, ¿terapéuticos?}

La literatura respecto a los efectos positivos del reposo en la salud mental es bastante escasa. Un estudio transversal realizado por Floderus con individuos a los que se les indicó un reposo prolongado (12-18 meses), por distintas enfermedades (no solamente psiquiátricas), mostró que si bien la gran mayoría tuvo consecuencias negativas, entre 16 y $31 \%$ se habría favorecido por esta indicación. Los efectos positivos fueron más frecuentes en mujeres y se relacionaron con mayor bienestar general en relación al sueño, estilo de vida, relaciones familiares y posibilidades de ocio. Se vio que el reposo en estos casos redujo el consumo de alcohol y tabaco, efecto ligeramente más notorio en hombres. Se concluye en este estudio que algunos pacientes encontrarían un bienestar al estar fuera de su trabajo, pero no necesariamente por el alejamiento que esto implica respecto a posibles estresores laborales ni tampoco a un factor terapéutico per se del reposo médico, sino a la exposición a factores protectores extralaborales inespecíficos de la salud mental ${ }^{25}$. Sin embargo, deberíamos preguntarnos si es la licencia el mé-
Tabla 2. Ejemplos validados de indicación de reposo médico en distintos sistemas de salud ${ }^{57-59}$

1. Permitir la realización de una intervención o procedimiento médico (e.g. intervenciones quirúrgicas)

2. Validar la discapacidad funcional transitoria que ciertas enfermedades provocan (e.g. en depresiones severas con alto grado de disfunción cognitiva y labilidad emocional)

3. Cuando per se es curativo (e.g. en la recuperación de una fractura o cirugía)

4. Promoción y prevención de la salud (i.e. reposo perinatal y permiso materno para enfermedades de hijo de hasta un año de edad [Chile])

5. Cuidado de familiares enfermos (e.g. en países como EE. UU. y Australia)

6. Duelo (e.g. en EE. UU.)

todo más adecuado para que el paciente acceda a este tipo de beneficios.

Otra manera de entender los posibles efectos positivos del reposo tiene relación con evitar el presentismo, es decir, ahorrarle al paciente los efectos negativos de trabajar cuando se está enfermo $^{26}$. En la literatura científica, el presentismo fue definido en un comienzo como el impacto económico de trabajar estando enfermo, debido a la pérdida en la productividad que esto implica. En esas circunstancias, la productividad se ve limitada debido a la incapacidad para cumplir las fechas límites, dificultades en la concentración y no ser capaz de pensar claramente, entre otros factores ${ }^{27}$.

El presentismo es un fenómeno frecuente en los ambientes laborales. Estudios han demostrado que entre $72 \%$ y $83 \%$ de los empleados han ido a trabajar pese a estar enfermos, al menos una vez durante el último año ${ }^{28}$. Además de los efectos económicos revisados, el presentismo laboral puede acarrear efectos deletéreos para la salud. Estos incluyen enfermedades cardiovasculares ${ }^{29}$, peor salud general ${ }^{30,31} \mathrm{e}$ incluso mayor probabilidad de ausentismo en el futuro ${ }^{32}$. Asimismo, el presentismo de más de 5 episodios durante un año aumenta el riesgo de una licencia médica prolongada (mayor a 30 días) durante el período de seguimiento ${ }^{33}$.

Lo anterior también se aplica a las condiciones de salud mental, ya que sabemos que la mayoría de los trabajadores con depresión se mantienen 
trabajando y alrededor de $60 \%$ de ellos reportan impedimentos relacionados con el rendimiento, principalmente síntomas cognitivos tales como: dificultades en la concentración, indecisión y fallas de memoria ${ }^{34}$.

En consecuencia, deberíamos preguntarnos, al extender una licencia médica, cuánto puede perder el individuo por el presentismo en contraposición a su ausentismo en cada condición particular. Por último, no contamos con evidencia que fundamente la indicación de reposo con un fin terapéutico. Más bien los datos expuestos apuntan a que el reposo podría justificarse desde la perspectiva del déficit funcional provocado por la enfermedad.

\section{Consecuencias negativas del reposo médico}

Las personas que están con reposo por enfermedad también presentan consecuencias negativas en distintas áreas de su vida (salud, proyección laboral, relaciones interpersonales y su economía), que son mayores de las atribuibles a la condición de salud que motivó el reposo ${ }^{35-37}$.

\section{a) Efectos psicológicos}

El reposo médico alivia el presentismo asociado a una condición psiquiátrica, pero con el costo de perder los beneficios psicológicos y sociales propios del trabajo, en el supuesto de que existan condiciones laborales suficientemente buenas. Jahoda los ha resumido así: proveer de un estatus personal e identidad social, promover la interacción social, vincular a los individuos con objetivos y propósitos colectivos que trascienden los propios y, además, generar una actividad regular y estructurada ${ }^{38}$. Esto es especialmente importante en condiciones como la depresión, ya que múltiples autores han demostrado que la inactividad y el retraimiento social contribuyen a exacerbar los componentes afectivos y cognitivos de la depresión ${ }^{39}$, generando un círculo vicioso que mantiene y profundiza la sintomatología.

Además, existen dos fenómenos que han sido estudiados cuando el reposo médico se prolonga. El primero es una disminución de la probabilidad de volver a trabajar ${ }^{40-42}$. Estudios demuestran que luego de doce semanas fuera del trabajo, el riesgo de ausentismo laboral prolongado aumenta drásticamente y a los seis meses de licencia médica una persona tiene solo $20 \%$ de probabilidades de estar trabajando en un plazo de 5 años $^{24}$. Otro estudio del Reino Unido demuestra que solo $10 \%$ de las personas que reciben un beneficio por incapacidad (habitualmente otorgado a las 26 semanas de licencia) se reintegrará laboralmente en el corto plazo $^{43}$. Lo segundo es que se puede desarrollar una fobia al lugar de trabajo, descrita como una reacción de pánico con arousal psicológico al pensar o aproximarse a este ${ }^{44}$. Es un fenómeno frecuente y además uno de los factores que más obstaculiza un retorno exitoso al trabajo ${ }^{45,46}$. Al igual que en otros trastornos de ansiedad y fobias, los pacientes desarrollan conductas evitativas, lo que lleva a mayor deterioro, ya que perpetúa un círculo vicioso de ansiedad y evitación.

\section{b) Efectos laborales y económicos}

Debido al constante aumento de las licencias psiquiátricas ha habido una mayor fiscalización de estas respecto a aquellas licencias por otros diagnósticos ${ }^{47}$. De hecho, las licencias por trastornos mentales son las que concentran el mayor número de rechazos, tanto en FONASA $(11,9 \%)$ como en el sistema privado (ISAPREs) $(38,1 \%)^{2}$. La tendencia, desde el 2004 en adelante, en relación al porcentaje de días pagados respecto a los días indicados por el médico, ha sido hacia la baja en este tipo de reposo ${ }^{48}$. Esto implica dificultades económicas para el paciente que serán un estresor adicional a su padecimiento psíquico. Además, esto ha perjudicado a aquellos pacientes que sí ameritan un reposo, ya que son sometidos a un mayor nivel de sospecha, tanto por parte de los tratantes como por entidades fiscalizadoras e incluso en su lugar de trabajo.

Para el desarrollo profesional, la licencia psiquiátrica también puede tener consecuencias negativas. El reposo prolongado (mayor a 28 días) tiene un efecto deletéreo en el salario a futuro ${ }^{49}$, en la posibilidad de hacer carrera o de cambiar a otro trabajo y, en ocasiones, conlleva una pérdida del interés en el mismo, incluso en los pacientes que se reintegran ${ }^{50,51}$.

\section{c) Mortalidad postrreposo médico prolongado}

Este aspecto es el más controversial, ya que la mortalidad es una medida en la que influye un amplio y complejo número de factores. Por tanto, para determinar una asociación entre reposo y mortalidad se hace necesario despejar variables que son comunes a ambos eventos, tanto sociodemográficas como médicas y laborales. Rigurosos 
estudios con estas características, realizados en cohortes de millones de pacientes en países del área escandinava e Inglaterra, han encontrado un aumento en las tasas de mortalidad general tras un período de reposo prolongado en el seguimiento a largo plazo (6 a 13 años postrreposo).

Al revisar el diagnóstico que fundamenta el reposo clínico, los cuadros psiquiátricos se correlacionan en todos los casos con un aumento de mortalidad posterior ${ }^{52-54}$. Un estudio en el Reino Unido $^{54}$ precisa que los cuadros psiquiátricos no psicóticos y neuróticos serían específicamente los que se relacionan a mayor muerte posterior a una licencia, tanto en la mortalidad general como en la asociada a cáncer, enfermedades cardiovasculares y suicidio.

En cuanto a la "dosis" del factor analizado (i.e. duración del reposo), en dos estudios ${ }^{53,54}$ se encuentra una correlación directa entre la dosis y mortalidad posterior. Esta asociación cae con el paso del tiempo desde el reposo índice ${ }^{18,21}$, salvo en un estudio ${ }^{55}$ en el que aquellos pacientes que presentaron reposos especialmente prolongados persistieron con tasas de mortalidad elevada más allá de 5 años posterior al período de reposo.

\section{Discusión}

Los cambios revisados acerca del reposo médico psiquiátrico nos permiten plantear que la consulta de salud mental le ha ido abriendo el paso progresivamente a problemáticas sociales en búsqueda de una solución concreta. Ante tal situación nos preguntamos: ¿es la consulta el lugar más adecuado para resolver estos problemas? Y si así fuera, ¿tenemos en la consulta los elementos necesarios para hacernos cargo de este tipo de factores? Por último, ¿es el reposo una respuesta médicamente válida?

La indicación de reposo nos sitúa a los médicos ante un cruce de dos conflictos: por un lado, sopesar los efectos positivos y negativos de la indicación del reposo en cada paciente y, por otro, sostener el doble rol que implica estar entremedio de los intereses del paciente y de la sociedad. Podríamos pensar el reposo médico como un fármaco cuyo mecanismo de acción desconocemos, así como a quiénes o por cuánto tiempo beneficia y que, al mismo tiempo, tiene efectos adversos conocidos como los revisados en este artículo. La situación es aún más compleja si consideramos que no hay directrices claras respecto a cómo indicar el reposo médico en psiquiatría.

Volviendo a las preguntas planteadas en el primer párrafo, surge una reflexión acerca de cuál es el rol que médicos y psiquiatras tienen ante pacientes que consultan por ciertas problemáticas sociales que, pudiendo ser válidas, no necesariamente se relacionan con alguna condición psiquiátrica. No es inhabitual, por ejemplo, la consulta de mujeres hacia el final de su reposo postnatal solicitando licencia médica. Anteriormente esto explicó un uso excesivo de reposo médico relacionado a un sobrediagnóstico de reflujo gastroesofágico del lactante, siendo esta una área hoy regularizada en Chile. El problema era que cada médico debía decidir cuántos meses de reposo postnatal eran deseables en aquellas mujeres que llevaban a sus hijos, sin que se produjese una decisión a nivel de políticas públicas que beneficiara a toda la población y no solo a los consultantes en base al caso a caso. Por cierto, esto cae en el relativismo de los criterios que tiene cada profesional, generando una situación potencialmente injusta.

Otro ejemplo habitual es la consulta de personas que están cuidando a un familiar enfermo y presentan una reacción adaptativa leve o incluso se encuentran asintomáticos, aunque el no poder hacerse cargo de dicha tarea pudiese implicar la agudización o aparición de síntomas. Nuevamente, el problema en un caso como este radica en que queda a criterio del médico decidir sobre asuntos que a nivel global el país no ha establecido cobertura. La interpretación de la severidad de los síntomas, así como de la validez del factor externo, resulta una tarea compleja y posiblemente arbitraria en situaciones como esta última. Pensamos que no es el médico el profesional, ni la consulta el lugar más indicado para ponderar y manejar estos factores extraclínicos.

Debemos considerar que la situación del trabajo en Chile es subóptima en diversas dimensiones, por lo tanto, es importante tener cautela al aplicar las conclusiones de trabajos realizados en países desarrollados a la realidad chilena. Esto nos podría llevar a la interpretación errónea de que el trabajo es un factor protector de la salud mental bajo cualquier circunstancia. Pensamos que la mejora de las condiciones laborales debiera producirse predominantemente a través de cambios a nivel de políticas laborales. 
Finalmente, queremos sugerir algunas recomendaciones que pudiesen hacerse a la luz de lo presentado. Primero es necesario que el clínico considere y pondere tanto los efectos positivos como potencialmente iatrogénicos del reposo y que haga partícipe al paciente de este proceso terapéutico desde el inicio. Esto facilitaría que el paciente se comprometiera con la minimización de los factores deletéreos que pudiera implicar el reposo y ayudaría con el proceso de reintegro laboral. A un nivel sistémico, nuestra propuesta es que los fenómenos que describimos en este trabajo estimulen a generar una reflexión que convoque a médicos y a otros profesionales que participan en salud a investigar en profundidad estos temas, en vista a proponer cambios a nivel de políticas públicas (e.g. formas de desincentivar el reposo prolongado: pago parcial de la licencia, estimular el reposo de tiempo parcial, otorgar días limitados para el cuidado de un familiar enfermo, etc), así como también incorporar esta temática en la docencia de pre y postgrado.

\section{Referencias}

1. Whiteford H, Ferrari A, Degenhardt L, Feigin V, Vos T. The global burden of mental, neurological and substance use disorders: an analysis from the global burden of disease study 2010. PLoS ONE 2015; 10 (2): e0116820.

2. Superintendencia de Salud. Análisis de Licencias Médicas y Gasto en Subsidios por Incapacidad Laboral 2011. Departamento de Estudios y Desarrollo, Chile, 2012. Disponible en: http://www.supersalud.gob.cl/ difusion/665/articles-7835_recurso_1.pdf [Consultado el 30 de abril de 2018].

3. Superintendencia de Seguridad Social. Análisis de Licencias Médicas por Enfermedades Mentales de Origen Común - año 2015. Chile, 2016. Disponible en: http:// www.suseso.cl/wp-content/uploads/2015/05/20161205_ Licencias_Medicas_Mentales_2016.pdf [Consultado el 10 de enero de 2017].

4. Marconi J. La psiquiatría en el cambio de siglo: psiquiatría social. Rev Chil Neuro-Psiquiat 2001; 39 (1): 10-1.

5. Alarcón RD, Aguilar-Gaxiola SA. Mental health policy developments in Latin America. Bull World Health Organ 2000; 78 (4): 483-90.

6. Minoletti A, Sepúlveda R, Horvitz-Lennon M. Twenty Years of Mental Health Policies in Chile. Int J Ment Health 2012; 41 (1): 21-37.

7. Sobocki P. The economic burden of depression in
Sweden from 1997 to 2005. Eur Psychiatry 2007; 22 (3): 146-52.

8. Botto A, Acuña J, Jiménez JP. La depresión como un diagnóstico complejo. Rev Med Chile 2014; 142 (10): 1297-305.

9. Botto A. Entre la diversidad y la confusión: algo huele mal en el estado depresivo. Rev GPU 2015; 11 (2): 1223.

10. Davidsen AS, Fosgerau CF. What is depression? Psychiatrists' and GPs' experiences of diagnosis and the diagnostic process. Int J Qual Stud Health Well-being 2014; 9 (Nov): 24866. Disponible en: http://dx.doi. org/10.3402/qhw.v9.24866 [Consultado el 3 de enero de 2017].

11. Neves Pinheiro da Costa S, Teixeira LH, Bezerra LN. Burnout at Work in Modern Times. J Clin Med Res 2015; 7 (10): 752-6.

12. OECD. OECD Employment Outlook 2015, OECD Publishing, Paris. Disponible en: http://doi.org/10.1787/ empl_outlook-2015-en [Consultado el 13 de enero de 2017].

13. Bermejo I, Kriston L, Schneider F, Gaebel W, Hegerl U, Berger M, et al. Sick leave and depression - Determining factors and clinical effect in outpatient care. Psychiatry Res 2010; 180 (2-3): 68-73.

14. Coe J. The physician's role in sickness absence certification: a reconsideration. J Occup Med 1975; 17 (11): 722-4.

15. Mayhew HE, Nordlund DJ. Absenteeism certification: the physician's role. J Fam Pract 1988; 26 (6): 651-5.

16. Edlund C, Dahlgren L. The physician's role in the vocational rehabilitation process. Disabil Rehabil 2002; 24 (14): 727-33.

17. Feeney A, North F, Head J, Canner R, Marmot M. Socioeconomic and sex differentials in reason for sickness absence from the Whitehall II Study. Occup Environ Med 1998; 55 (2): 91-8.

18. Timpka T, Hensing G, Alexanderson K. Dilemmas in sickness certification among Swedish physicians. Eur J Public Health 1995; 5 (3): 215-9.

19. Murray TH. Divided loyalties for physicians: social context and moral problems. Soc Sci Med 1986; 23 (8): 827-32.

20. Wahlström R, Alexanderson K. Physicians' sick-listing practices. Scand J Public Health 2004; 32 (Suppl 63): 222-55.

21. Alexanderson K, Norlund A (Eds). Sickness absencecauses, consequences and physicians' sickness certification practice. A systematic literature review by the Swedish Council on Technology Assessment in Health Care. Scand J Public Health 2004; 32 (Suppl 63): 1-263. 
22. Hiscock J, Ritchie J. The role of GPs in sickness certification. Research Report No 148. Leeds: Department for Work and Pensions, 2001.

23. Norrmén G, Svärdsudd K, Andersson DKG. How primary health care physicians make sick listing decisions: the impact of medical factors and functioning. BMC family practice 2008; 9 (1): 3. Disponible en: http://bmcfampract.biomedcentral.com/articles/10.1186/1471-2296-9-3 [Consultado el 3 de enero de 2017].

24. Dunstan D. Are sickness certificates doing our patients harm? Aust Fam Physician 2009; 38 (1/2): 61-3.

25. Floderus B, Göranson S, Alexanderson K, Aronsson G. Self-estimated life situation in patients on long-term sick leave. J Rehabil Med 2005; 37: 291-9.

26. Sanderson K, Cocker F. Presenteeism-implications and health risks. Aust Fam Physician 2013; 42 (4): 172-5.

27. Lerner D, Adler D, Chang H, Lapitsky L, Hood M, Perissinotto C, et al. Unemployment, Job Retention, and Productivity Loss Among Employees With Depression. Psychiatr Serv 2004; 5 (12): 1371-8.

28. Elstad JI, Vabo M. Job stress, sickness absence and sickness presenteeism in Nordic elderly care. Scand J Public Health 2008; 36: 467-74.

29. Kivimäki M, Head J, Ferrie J, Hemingway H, Shipley $\mathrm{M}$, Vahtera J, et al. Working while iII as a risk factor for serious coronary events: the whitehall II study. Am J Public Health 2005; 95 (1): 98-102.

30. Gustafsson K, Marklund S. Consequences of sickness presence and sickness absence on health and work ability: A swedish prospective cohort study. Int J Occup Med Environ Health 2011; 24 (2): 153-65.

31. Bergstrom G, Bodin L, Hagberg J, Lindh T, Aronsson G, Josephson M. Does sickness presenteeism have an impact of future general health?. Int Arch Occup Environ Health 2009; 82 (10): 1179-90.

32. Hansen CD, Andersen JH. Going ill to work-what personal circumstances, attitudes and work-related factors are associated with sickness presenteeism?. Soc Sci Med 2008; 67: 956-64.

33. Bergstrom G, Bodin L, Hagber J, Aronsson G, Josephson M. Sickness presenteeism today, sickness absenteeism tomorrow? A prospective study on sickness presenteeism and future sickness absenteeism. J Occup Environ Med 2009; 51 (6): 629-38.

34. Wang YP, Gorenstein C. Attitude and impact of perceived depression in the work place. Int J Environ Res Public Health 2014; 11: 6021-36.

35. SBU [Swedish Council on Technology Assessment and Health Care]. Sickness absence - causes, consequences, and physicians' sickness certification practice. A sys- tematic literature review by the Swedish Council on Technology Assessment and Health Care. Scand J Public Health 2004; 32: 3-263.

36. Bryngelson A. Long-term sickness absence and social exclusion. Scand J Public Healt 2009; 37 (8): 839-45.

37. Eriksson H-G, von Celsing A-S, Wahlström R, Janson L, Zander V, Wallman T. Sickness absence and self-reported health a population-based study of 43,600 individuals in central Sweden. BMC Public Health 2008; 8: 426. Disponible en: http://bmcpublichealth.biomedcentral.com/articles/10.1186/1471-2458-8-426 [Consultado el 6 de octubre de 2016].

38. Jahoda, M. Work, employment and unemployment: Values, theories and approaches in social research. Am Psychol 1981; 36:184-91.

39. Meichenbaum D, Gilmore JB. La naturaleza de los procesos inconscientes: una perspectiva cognitivo-conductual. Revista terapia psicológica 1984; Año III, Nº4: 7-22.

40. Wallman T, Wedel H, Palmer E, Rosengren A, Johansson S, Eriksson $\mathrm{H}$, et al. Sick-leave track record and other potential predictors of a disability pension. A population based study of 8,218 men and women followed for 16 years. BMC Public Health 2009, 9: 104. Disponible en: http://bmcpublichealth.biomedcentral. com/articles/10.1186/1471-2458-9-104 [Consultado el 6 de octubre de 2016].

41. Hultin H, Lindholm C, Malfert M, Möller J. Short-term sick leave and future risk of sickness absence and unemployment - the impact of health status. BMC Public Health 2012; 12: 861. Disponible en: http://bmcpublichealth. biomedcentral.com/articles/10.1186/1471-2458-12-861 [Consultado el 6 de octubre de 2016].

42. Virtanen M, Kivimäki M, Vahtera J, Elovainio M, Sund $\mathrm{R}$, Virtanen $\mathrm{P}$, et al. Sickness absence as a risk factor for job termination, unemployment, and disability pension among temporary and permanent employees. Occup Environ Med 2006; 63: 212-7.

43. Sawney P. Current issues in fitness for work certification. Br J Gen Pract 2002; 52 (576): 217-22.

44. Muschalla B, Linden M. Workplace phobia - a first explorative study on its relation to established anxiety disorders, sick leave, and work-directed treatment. Psychol Health Med 2009; 14 (5): 591-605.

45. Yarker J, Munir F, Donaldson-Feilder E, Hicks B. Managing rehabilitation: a competency framework for managers to support return to work. London: British Occupational Health Research Foundation, 2010.

46. Muschalla B, Linden M, Olbrich D. The relationship between job-anxiety and trait-anxiety - A differential diagnostic investigation with the Job-Anxiety-Scale and 
the State-Trait-Anxiety-Inventory. J Anxiety Disor 2010; 24 (3): 366-71.

47. Miranda G, Alvarado S, Kaufman J. Duración de las licencias médicas FONASA por trastornos mentales y del comportamiento. Rev Med Chile 2012; 140: $207-$ 13.

48. Ministerio de Salud. Guía Referencial: Reposo médico y reintegración laboral en personas con problemas y/o enfermedad mental. 2010, Chile. Disponible en: http:// www.cetep.cl/web/wp-content/uploads/2011/08/Presentaci\%C3\%B3n-Dr.-Alfredo-Pemjean.pdf [Consultado el 30 de abril de 2018].

49. Andersen SH. The cost of sickness: On the effect of the duration of sick leave on post-leave earnings. Soc Sci Med 2010; 70: 1581-9.

50. Sieurin L, Josephson M, Vingard E. Positive and negative consequences of sick leave for the individual, with special focus on part-time sick leave. Scand J Public Health 2009; 37 (1): 50-6.

51. Judiesch M, Lyness K: Left behind? The impact of leaves of absence on managers' career success. Acad Managem J 1999; 42 (6): 641-51.

52. Bryngelson A, Åsberg M, Nygren Å, Jensen I, Mittendorfer-Rutz E. All-Cause and Cause-Specific Mortality after Long-Term Sickness Absence for Psychiatric Disorders: A Prospective Cohort Study. PLoS ONE 2013; 8(6): e67887. Disponible en: http://journals.plos.org/plosone/ article?id=10.1371/journal.pone.0067887 [Consultado el 6 de octubre de 2016].

53. Wang M, Alexanderson K, Runeson B, Head J, Melchior $\mathrm{M}$, Perski A, et al. Are all-cause and diagnosis-specific sickness absence, and sick-leave duration risk indicators for suicidal behaviour? A nationwide register-based cohort study of 4.9 million inhabitants of Sweden. Occup Environ Med 2014; 71 (1): 12-20.
54. Head J, Ferrie J, Alexanderson K, Westerlund H, Vahtera J, Kivimaki M. 'Diagnosis-specific sickness absence as a predictor of mortality: the Whitehall II prospective cohort study'. BMJ 2008; 337:a1469. Disponible en: http:// www.bmj.com/content/337/bmj.a1469 [Consultado el 6 de octubre de 2016].

55. Vahtera J, Pentti J, Kivimaki M. Sickness absence as a predictor of mortality among male and female employees. J Epidemiol Community Health 2004; 58 (4): 321-6.

56. Escuela de Medicina Universidad Católica de Chile. Informe final estudio de carga de enfermedad y carga atribuible. Departamento de Salud Pública, encargado por Ministerio de Salud de Chile, 2008. Disponible en: http://www.cienciasdelasalud-udla.cl/portales/ tp76246caadc23/uploadImg/File/Informe-final-carga-Enf-2007.pdf [Consultado el 30 de abril de 2018].

57. US Office of Personnel Management. Fact Sheet: Sick Leave to Care for a Family Member with a Serious Health Condition. Disponible en: https://www.opm.gov/ policy-data-oversight/pay-leave/leave-administration/ fact-sheets/sick-leave-to-care-for-a-family-memberwith-a-serious-health-condition/ [Consultado el 11 de julio de 2017].

58. Fair Work Ombudsman, Australian Government. Notice \& medical certificates. Disponible en: https:// www.fairwork.gov.au/leave/sick-and-carers-leave/paidsick-and-carers-leave/notice-and-medical-certificates [Consultado el 11 de julio de 2017].

59. US Office of Personnel Management. Fact Sheet: Sick Leave for Family Care or Bereavement Purposes. Disponible en: https:/www.opm.gov/policy-data-oversight/ pay-leave/leave-administration/fact-sheets/sick-leave-for-family-care-or-bereavement-purposes/ [Consultado el 11 de julio de 2017]. 